\title{
Dose-Response Relation between Exercise Duration and Cognition
}

By: Chang, Yu-Kai, Chu, Chien-Heng, Wang, Chun-Chih, Wang, Yi-Chun, Song, Tai-Fen, Tsai, Chia-Liang, Etnier, Jennifer L.

This is a non-final version of an article published in final form in Chang, Y.K., Chu, C.H., Wang, C.C., Wang, Y.C., Song, T.F., Tsai, C.L., \& Etnier, J.L. (2015). Dose-response relationship between exercise duration and cognition. Medicine and Science in Sports and Exercise, 47(1), 159-165

Made available courtesy of American College of Sports Medicine: http://journals.lww.com/acsm-msse/pages/default.aspx

\section{Abstract:}

Purpose: The study aimed to provide evidence-based recommendations for the prescription of a single session of exercise to improve cognitive performance. In particular, the purpose was to determine the dose-response relation between exercise duration and cognitive performance for a moderate-intensity session of aerobic exercise.

Methods: Twenty-six healthy young men participated in a reading control treatment and three exercise treatments presented in a random order. The exercise treatments were designed on the basis of the American College of Sports Medicine guidelines and consisted of a 5-min warm-up, a 5-min cooldown, and cycling at moderate intensity (approximately 65\% HR reserve) for 10, 20, or $45 \mathrm{~min}$. The Stroop test was administrated after completion of each assigned treatment.

Results: Exercise at moderate intensity for 20 min resulted in significantly better cognitive performance, as assessed by shorter response time and higher accuracy. This result was found regardless of the type of cognitive function assessed. In addition, a curvilinear dose-response relation between exercise duration and cognitive performance was observed.

Conclusions: An exercise session consisting of a 5-min warm-up, 20 min of moderate-intensity exercise, and a 5-min cooldown improves cognition, whereas shorter or longer durations of moderate exercise have negligible benefits. This study provides the foundation for the prescription of a single session of moderate exercise to facilitate cognitive function in healthy younger adults.

Keywords: Cognitive performance | Executive function | Inhibition | Inverted U hypothesis | Stroop test 


\section{Article:}

Substantial literature has demonstrated a positive effect of a single bout of exercise on cognitive performance $(9,27,35)$. Specifically, the results of two meta-analyses indicate that acute exercise produces a small, significant, positive effect on cognitive performance performed after the exercise session (Chang et al. (9): effect size (ES), 0.11 for immediately after, and ES, 0.10 for after a delay; Lambourne and Tomporowski (27): ES, 0.20 for after exercise). Given this facilitative effect on cognitive performance, one important direction for research is to explore dose-response characteristics to define the optimal level of acute exercise with regard to cognitive benefits $(9,27)$. This is important because an understanding of dose-response relations will provide the foundation from which to establish exercise prescription to facilitate cognitive performance.

Exercise-induced change in arousal is one mechanism that has been proposed to underlie the relation between acute exercise and cognition. A large number of studies have focused on understanding how variations in physiological arousal induced by different exercise intensities influence the effects on cognitive performance. However, much of this research has examined the influence on cognitive performance, as assessed during exercise rather than after exercise. Some of the studies exploring the effects during exercise demonstrate a linear dose-response trend, such that increased exercise intensity is directly related to improved cognitive performance $(8,15,31)$. Other researchers have reported a curvilinear trend, also described as an inverted $\mathrm{U}$ relation, indicating that moderate exercise intensity results in the best cognitive performance as compared with low and vigorous exercise intensities $(2,12)$. In contrast, there are only a few studies in which the dose-response relation between aerobic exercise intensity and cognitive performance performed after exercise has been assessed. Results of these studies support an inverted $U$ relation, such that neuroelectric indices of cognition were best at moderate intensity and not as good at lower or higher intensities (24-26). Differences in findings across these studies may be due to myriad factors including the timing of the cognitive assessment and the particular cognitive task assessed. Interestingly, two studies have explored the dose-response relation between resistance exercise intensity and cognitive performance after exercise and indicated that the nature of the relation between exercise intensity and performance is specific to a task. Specifically, Chang et al. $(6,7)$ found that resistance exercise performed at $100 \%$ of 10 repetition maximum (RM) facilitated lower-level cognitive function relative to $40 \%$ and $70 \%$ of 10RM, suggesting a linear trend, whereas $70 \%$ of 10RM resulted in better performance on interference and working memory (7) and planning (6) aspects of executive function than did either $100 \%$ or $40 \%$ of $10 \mathrm{RM}$, reflecting an inverted $U$ trend. Thus, it is important for future studies to consider the potential task specificity of dose-response relations between acute exercise intensity and cognitive performance.

It is worth noting that an exercise prescription for acute exercise involves intensity, modality, and duration (1), and despite relatively well-described dose-response relations between exercise intensity and cognitive function, the effect of exercise duration has not yet been examined. In a narrative review, Tomporowski (34) proposed that submaximal exercise performed for 20-60 
min would benefit cognitive performance. However, this conclusion was based on studies that generally emphasized lower-level cognitive performance, and hence, the results may not apply to higher-order measures such as measures of executive function. To date, the effect of exercise duration on higher-order functions can only be considered indirectly by comparing across results from studies that have looked at a single duration (e.g., 10, 30, $120 \mathrm{~min}$ ), and when examined this way, results are ambiguous. For instance, whereas some studies indicate that moderateintensity exercise performed for 20-30 min has a positive effect on executive function, exercise for less than 20 min or more than 30 min has resulted in facilitation, no effect, or even impairment on executive function tasks $(3,13,14,23)$. These conflicting results emphasize the importance of studying the effects of exercise duration on cognition in a single study, with the long-range goal being to inform exercise prescription. When reviewed meta-analytically, results for studies testing the effects of acute exercise on cognitive performance performed after the cessation of exercise indicate that the exercise must last at least $11 \mathrm{~min}$ to result in a positive effect (9). However, because these results rely on averaging effects across studies, this conclusion may be influenced by other variables that differ between studies.

As such, the aim of the present study was to test the dose-response relation between exercise duration and cognitive function in a single study. In addition, the study was designed to consider the potential moderating effect of cognitive task type. While several cognitive tasks have been used in studies testing the effects of acute exercise on cognition (e.g., flanker task, go/no go test, and Tower of London) $(6,21,26)$, the Stroop test was particularly chosen because it is one of the most commonly used neuropsychological assessments to measure executive function (16). The Stroop test is also sensitive to acute exercise and consists of two tasks, which allow for the assessment of basic information processing and higher-order aspects of cognition $(10,16)$. Considering previous studies, we hypothesized that exercise performed at moderate intensity for 20 min would facilitate performance on both of the Stroop tasks whereas exercise performed for 10 or 45 min would have limited positive effects.

\section{METHODS}

\section{Participants}

Twenty-six young male adults between the ages of 20 and $22 \mathrm{yr}$ from two universities surrounding Taoyuan County, Taiwan, were recruited via flyers or referral from participants previously included in the study. Participants provided a written informed consent to participate after being provided with information regarding the potential risks and discomforts related to the study. The project was approved by the institutional review board of National Taiwan University. All participants met the inclusion criteria, as determined from the health screening questionnaire (e.g., participants had to be categorized as low risk) and the Physical Activity Readiness Questionnaire (participants had to answer "no" for all items). These questionnaires were reviewed following the guidelines of the American College of Sports Medicine (ACSM) (1) to ensure the safety of the participants performing the cardiovascular fitness test and acute 
bouts of exercise. In addition, all of the participants were right hand dominant and none reported any neurological or psychiatric disorders, color blindness, medical conditions, or abnormal vision that might influence performance of the cognitive tasks. Eligible participants were required to complete the International Physical Activity Questionnaire (IPAQ), an international surveillance measure assessing physical activity participation $(4,29)$, and the digit span test of the Wechsler Adult Intelligence Scale (36) to describe other variables that may influence cognitive performance. The sample size was determined using power analysis in which a one-way ANOVA with ES partial eta squared ([eta]2) of 0.42 , power of 0.8 , and alpha at 0.05 were used (11). Participants' demographic characteristics are presented in Table 1.

\begin{tabular}{lc}
\hline Variable & Total \\
\hline Sample size & 26 \\
Age $(\mathrm{yr})$ & $20.77 \pm 0.91$ \\
Education $(\mathrm{yr})$ & $14.77 \pm 0.91$ \\
Height $(\mathrm{cm})$ & $174.73 \pm 6.09$ \\
Weight $(\mathrm{kg})$ & $67.77 \pm 10.08$ \\
BMI $\left(\mathrm{kg} \cdot \mathrm{m}^{-2}\right)$ & $22.04 \pm 2.68$ \\
IPAQ $\left(\mathrm{MET} \cdot \mathrm{wk}^{-1}\right)$ & $3800.23 \pm 437.2$ \\
VO $\mathrm{O}_{2 \text { peak }}\left(\mathrm{mL}^{-1} \cdot \mathrm{kg}^{-1} \cdot \mathrm{min}^{-1}\right)$ & $42.45 \pm 6.49$ \\
Digit span forward & $14.00 \pm 1.47$ \\
Digit span backward & $9.19 \pm 2.28$ \\
\hline
\end{tabular}

BMI, body mass index.

TABLE 1 Participants' demographic characteristics (mean \pm 1 SD).

\section{Cardiovascular Fitness Test}

Participants performed a submaximal exercise test on an electronically braked cycle ergometer (Ergoselect 100/200; Ergoline GmbH, Germany) to indirectly determine their V.O2peak. This test was conducted so that fitness levels could be reported for the participants. The exercise test was the adapted YMCA cycle ergometry protocol recommended by the ACSM $(1,18)$. The YMCA protocol involves 2-4 consecutive 3-min cycling stages that are intended to elevate HR to a target zone of between $110 \mathrm{bpm}$ and $70 \%$ of the HR reserve (HRR) (i.e., resting HR plus $70 \%$ of the difference between age-predicted HRmax and resting HR) for two consecutive stages. The initial workload was $150 \mathrm{kpm} \cdot \mathrm{min}-1$ or $0.5 \mathrm{~kg}$ with a pedaling speed of $50 \mathrm{rpm}$. The experimenter recorded the participant's HR in the last $15-30 \mathrm{~s}$ of the second and third minutes, and the average of these two HR values was used to determine the workload for the next stage after the YMCA protocol (see ACSM guidelines, Ninth Edition (1), page 82). Once the HR reached the target HR zone for two consecutive stages, the test was terminated and V.O2peak was calculated on the basis of age-predicted HRmax, body mass, and a line depicting the relation between HR and power output during the final two stages.

Exercise Intensity Measurement 
HR

HR provides an objective index of exercise intensity. Thus, HR monitors (Sport Tester PE 3000; Polar Electro Oy, Kempele, Finland) were used to confirm the exercise intensity manipulation. The HR monitor was worn by participants throughout the treatment conditions, and HR was recorded by the experimenter at 2-min intervals. Two HR indices were identified: resting HR represents HR assessed after sitting quietly in a comfortable chair for 15 min before the treatment, whereas treatment HR represents HR during the treatment.

\section{RPE}

Ratings of perceived exertion (RPE) provide a subjective measurement of an individual's perception of effort during exercise (5). The Borg scale ranges from 6 to 20. RPE was measured at 2-min intervals during the moderate exercise portion of the exercise treatments.

\section{Cognitive Task: Stroop Test}

The Stroop test, also known as the Stroop Color-Word test, is a popular neuropsychological assessment used to measure a variety of cognitive functions and is sensitive to effects from an acute bout of exercise (10). Typically, the stimulus of the Stroop test is a color name that is printed in a different color of ink and the participant is instructed to name the color of the ink. The present study employed a modified computerized Stroop test administered using the Stim2 software (Neurosoft Labs, Inc., Sterling, VA), which allows for the assessment of reaction time and accuracy. This task consisted of two conditions. The congruent condition included three Chinese color words (i.e., for green, for blue, and for red) that were displayed in the same color (e.g., "green" printed in green ink), whereas the incongruent condition included the same three color names but each was displayed in a different color (e.g., "green" displayed in blue or red).

Each trial consisted of a fixed cross displayed on the center of the screen followed by a stimulus that was displayed for $506 \mathrm{~ms}$. Participants performed six blocks of 60 trials consisting of congruent trials (trials, $\mathrm{n}=38$ ) and incongruent trials (trials, $\mathrm{n}=22$ ) presented in a random order. The interval between the fixed cross and the presentation of the stimulus was randomly chosen for each trial as either 383, 583, or $783 \mathrm{~ms}$ to avoid stimulus expectation. Each stimulus was a 2$\mathrm{cm}$ square displayed on the center of the computer screen. The participant was required to identify the "color" of the target stimulus by pressing one of three labeled buttons with their right thumb on a response pane $(10 \times 8 \times 2 \mathrm{~cm}$ box $)$ as quickly and accurately as possible. With participants seated at approximately $70 \mathrm{~cm}$ from the computer screen, the participant's visual angle of the stimulus was $2^{\circ}$. An acceptable response was considered one that occurred within 200 and $1000 \mathrm{~ms}$ of the presentation of the stimulus. Responses that were not within the time range (i.e., 200-1000 ms) and responses when the participant pressed the wrong color button were considered inaccurate responses (Fig. 2 shows the percentages of accurate and inaccurate responses). Participants performed six blocks with a 2-min rest interval between each block (360 target trials in total), resulting in approximately $30 \mathrm{~min}$ for cognitive testing. Outcome variables were the average response time and accuracy rate for congruent and incongruent conditions. 


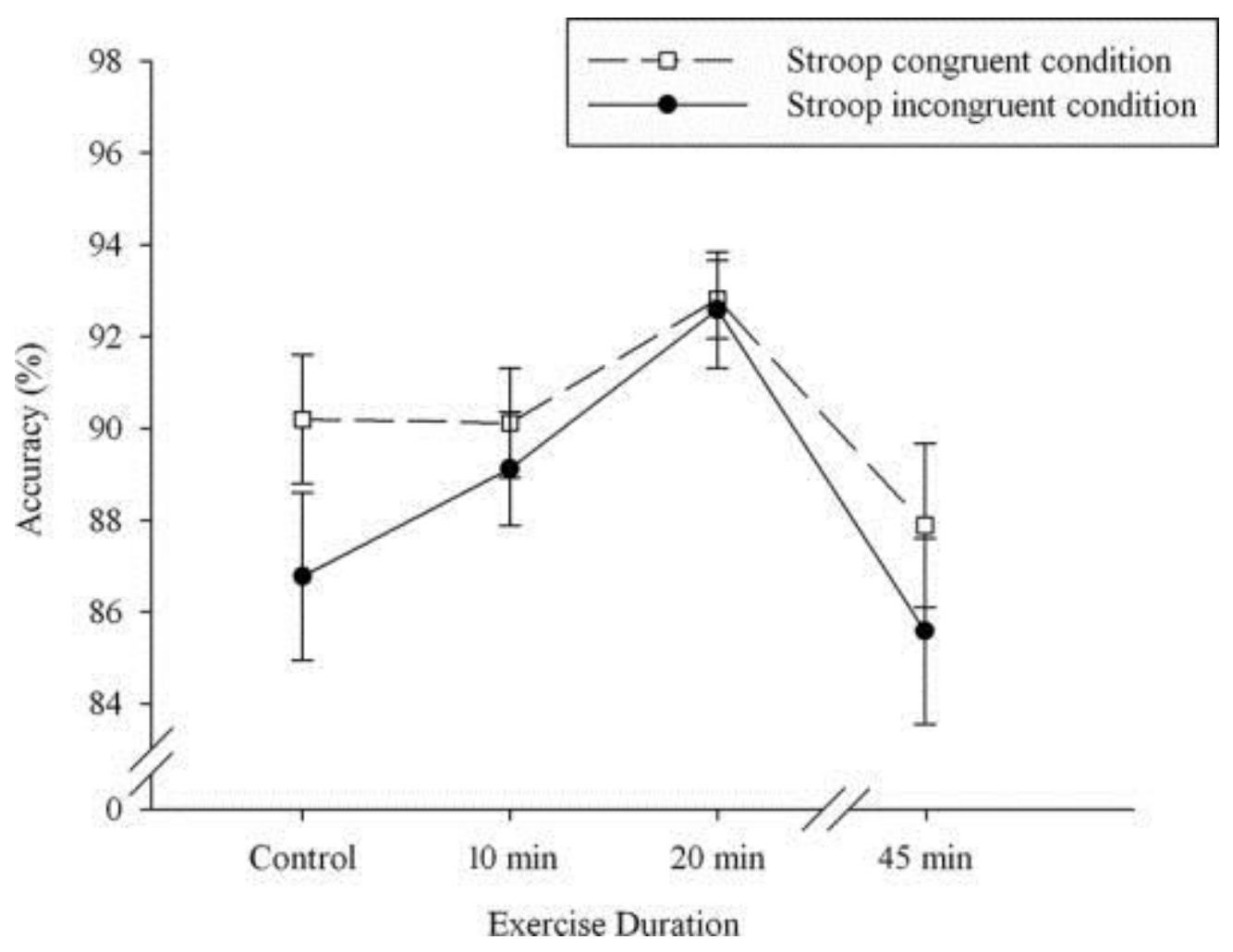

FIGURE 2. Dose-response relation between exercise duration and Stroop test performance in accuracy. Note that higher scores for accuracy are indicative of better performance. Values are presented as mean \pm SEM.

Treatments

In the reading treatment, participants were asked to read a book related to exercise and cognition for $30 \mathrm{~min}$. Immediately after this 30-min period, participants performed the Stroop task. After performing the Stroop task, participants performed the submaximal exercise test to assess cardiovascular fitness for descriptive purposes.

In the exercise treatments, participants were asked to perform an acute bout of steady-state exercise on a stationary cycle ergometer at $65 \% \mathrm{HRR}$ and a pedal rate of $65 \mathrm{rpm}$. The percentage of HRR was selected because this is considered to be of moderate intensity (1), and moderate intensity has been shown to be an appropriate intensity at which to observe cognitive performance benefits (11). The exercise durations were 10, 20, or 45 min with additional warmup and cooldown stages for 5 min each. The inclusion of a 5-min warm-up and a 5-min cooldown was based on the recommendations of the ACSM (1). In all treatments, the room temperature was $23^{\circ} \mathrm{C}$ and the humidity was between $40 \%$ and $60 \%$.

Procedures

Each participant was requested to come to the laboratory individually on four occasions with at least $3 \mathrm{~d}$ between each occasion. On these days, the participant received one of the four possible 
treatments (i.e., reading for $30 \mathrm{~min}$ or exercise for 10 , 20, or $45 \mathrm{~min}$ ) presented in a randomized counterbalanced order.

On the first visit, each participant was asked to complete the demographic questionnaire, the health screening questionnaire, and the Physical Activity Readiness Questionnaire. Eligible participants were then asked to complete the IPAQ and perform the digit span test. Then, each participant was instructed to perform practice trials on the Stroop test until at least an $85 \%$ accuracy rate was met. From this point on during the first visit, procedures were the same as those at every subsequent visit. At every visit, participants sat quietly for $15 \mathrm{~min}$, after which, a measure of resting HR was recorded. The participant then performed the assigned treatment for that session. After completing the assigned treatment (e.g., reading or exercise), participants were asked to complete the Stroop test within 5 min of cessation of treatment.

\section{Statistical Analyses}

Descriptive data are presented as mean $\pm \mathrm{SD}$, and three sets of analyses were conducted. To confirm the effectiveness of the exercise intensity manipulation, a $2 \times 4$ repeated-measures ANOVA (time: resting HR and treatment HR; treatment: reading and 10, 20, and 45 min) was conducted for the HR data. Two $4 \times 2$ (treatment $\times$ Stroop test condition) repeated-measures ANOVAs were then used to detect differences in response time and accuracy of performance on the Stroop test. Lastly, trend analyses were conducted to address the dose-response trend between exercise duration and Stroop test performance in terms of response time and accuracy. If the sphericity assumption was not met, Greenhouse-Geisser corrections. were used. Paired post hoc t-tests with Bonferroni adjustments for multiple comparisons were performed to follow up significant effects, ES of partial [eta] 2 were reported for significant effects, and an alpha of 0.05 was used for the significance level.

\section{RESULTS}

Exercise intensity manipulation check

Table 2 summarizes the descriptive data for HR and RPE relative to treatment condition. RPE during all exercise treatments ranged between 12 and 15, confirming that the exercise sessions were perceived as being at moderate intensity.

\begin{tabular}{|c|c|c|c|c|}
\hline \multirow[b]{2}{*}{ Varisbis } & \multicolumn{4}{|c|}{ Tneatneat } \\
\hline & Control & 10 mis & 20 mit & $45 \mathrm{~min}$ \\
\hline $\begin{array}{l}\text { Rusting HR (tope) } \\
\text { Tieatment HR (bpmi }\end{array}$ & $\begin{array}{l}72.39+7.07 \\
72.80+6.64\end{array}$ & $\begin{array}{r}20.62+9.35 \\
150.56+4.67\end{array}$ & $\begin{array}{r}70.62+8.35 \\
152.45=4.6 a\end{array}$ & $\begin{array}{r}71.00+9.36 \\
153.84+4.90\end{array}$ \\
\hline S HAR & NA & 65 & 66 & 67 \\
\hline RPE & NA & $12.65 \div 1.96$ & $13,47: 181$ & $14.52 \div 1.78$ \\
\hline
\end{tabular}

Nh not applabie.

TABLE 2 Descriptive data for exercise manipulation check and Stroop test performance (mean \pm 1 SD). 
A $2 \times 4$ repeated-measures ANOVA revealed that there were significant main effects for time $(\mathrm{F} 1,75=6067.33, \mathrm{P}<0.001$, partial $[\mathrm{eta}] 2=0.99)$ and treatment $(\mathrm{F} 3,75=780.54, \mathrm{P}<0.001$, partial [eta] $2=0.97)$. These significant main effects were superseded by a significant interaction between treatment and time $(\mathrm{F} 3,75=2748.12, \mathrm{P}<0.001$, partial [eta] $2=0.99)$. Follow-up multiple comparisons indicated that although no significant difference in resting HR existed among the four treatments, HR during the treatment was significantly higher during the three exercise treatments than during reading ( $\mathrm{P}$ values $<0.001)$. As expected, there were no significant differences among the three exercise durations and the average observed HR indicated that the exercise sessions were of moderate intensity (i.e., 65\%-67\% HRR) (Table 2).

\section{Stroop test performance}

Table 2 summarizes the descriptive data for response time and accuracy relative to treatment and Stroop test condition.

Regarding response time, results revealed a significant main effect of treatment $(\mathrm{F} 1,75=4.78, \mathrm{P}$ $<0.01$, partial [eta] $2=0.16)$ and Stroop test condition $(\mathrm{F} 1,75=60.63, \mathrm{P}<0.001$, partial [eta] $2=$ 0.71 ) but no interaction effect between treatment and Stroop test condition. Multiple follow-up comparisons for the treatment main effect revealed that response times were significantly shorter after the 20-min exercise session as compared with those after reading, 10 min of exercise, and 45 min of exercise $(\mathrm{P}<0.02)$ and that response times after reading and 10 and $45 \mathrm{~min}$ of exercise were not significantly different from one another $(\mathrm{P}>0.05)$. The results of the trend analysis support the finding by indicating a significant curvilinear trend $(\mathrm{P}<0.01$, partial [eta] $2=0.39)$ but not a significant linear trend $(\mathrm{P}>0.05)$. The main effect for the Stroop test condition indicated that longer response times were found for the incongruent condition compared with those for the congruent condition. Figure 1 displays the curvilinear effect of treatment and the main effect of the Stroop test condition on response time.

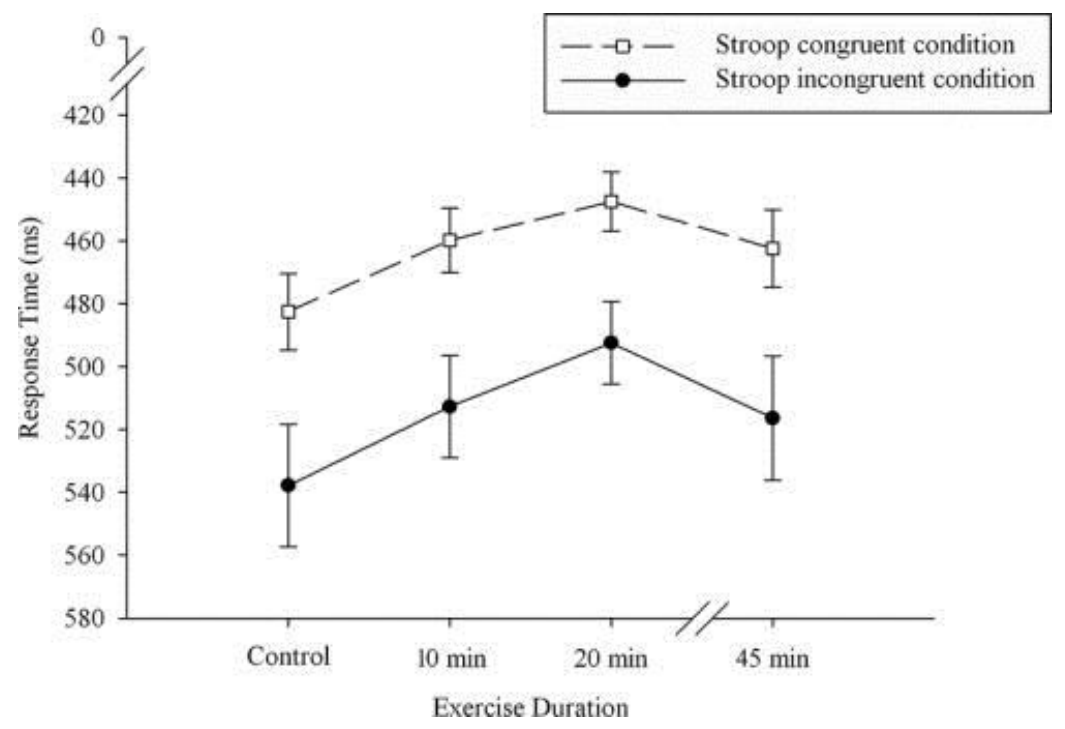

FIGURE 1. Dose-response relation between exercise duration and Stroop test performance in response time. Note that lower scores for response time are indicative of better performance. Values are presented as mean \pm SEM. 
Similar results were found in accuracy, where there were significant main effects for treatment $(\mathrm{F} 3,75=7.16, \mathrm{P}<0.001$, partial [eta] $2=0.22)$ and Stroop test condition $(\mathrm{F} 1,75=4.2, \mathrm{P}<0.05$, partial [eta] $2=0.14$ ) but no interaction effect between treatment and Stroop test condition. Multiple follow-up comparisons for the treatment main effect showed that accuracy was significantly better after $20 \mathrm{~min}$ of exercise as compared with that after the other three treatments $(\mathrm{P}<0.01)$, which were not significantly different from one another $(\mathrm{P}>0.05)$. Results of the trend analysis indicate a significant curvilinear trend $(\mathrm{P}<0.001$, partial [eta] $2=0.41)$ but no linear trend $(\mathrm{P}>0.05)$. Results for the main effect for the Stroop test condition indicated that higher accuracy rates were found for the congruent condition compared with those for the incongruent condition. Figure 2 displays the curvilinear effect of treatment and the main effect of the Stroop test condition on accuracy.

\section{DISCUSSION}

Acute bouts of exercise are thought to result in improved cognitive function, and several studies have investigated the dose-response relation between exercise intensity and cognitive performance after exercise. However, empirical studies have not been designed to test the effects of varying durations of acute exercise on cognitive performance, and therefore, the purpose of this study was to examine the effects of various durations of moderate-intensity aerobic exercise on cognitive performance after exercise. The results of this study showed that when exercising at moderate exercise intensity, the duration of the exercise affects both accuracy and response time on the Stroop task. The main finding was that exercising for $20 \mathrm{~min}$ resulted in shorter response time and higher accuracy compared with that in no exercise (reading control) and with exercise conducted for 10 or $45 \mathrm{~min}$, suggesting that there is a curvilinear relation between exercise duration and cognitive performance. In addition, because there was not a significant interaction between the Stroop test condition and treatment, it can be concluded that acute moderateintensity exercise positively affected performance regardless of the type of cognitive function assessed.

Our finding relative to the main effect for Stroop test condition replicates previous research that demonstrates the "Stroop effect"; that is, the incongruent condition resulted in longer response times and less accuracy relative to the congruent condition (30). In addition, we observed that moderate-intensity exercise performed for $20 \mathrm{~min}$ elicited the best performance in both incongruent and congruent conditions, suggesting that this duration of acute aerobic exercise at moderate intensity benefits lower- and higher-order aspects of cognition in general. The finding somewhat agrees with that in a previous study by Chang et al. (10) indicating general improvements in five different Stroop test conditions after acute exercise although larger benefits 
in the incongruent condition were also revealed. Importantly, the findings of this study suggest that moderate exercise intensity for $20 \mathrm{~min}$, with a 5-min warm-up and cooldown as recommended by the ACSM, results in the largest benefits to cognitive performance.

One hypothetical mechanism that has been proposed to explain the facilitative effect of 20 min of acute moderate-intensity exercise on cognitive performance is that after the exercise, greater attentional allocation, more efficient information processing speed, and optimal physiological arousal occurs. This interpretation is supported by studies applying neuroelectric techniques $(21,22,24,26)$. For example, Hillman et al. (22) observed that exercise at a moderate-intensity level elicits larger P3 amplitude and shorter P3 latency during a flanker task performed after the exercise. The authors interpreted this finding as indicative of an individual being able to devote more attentional resources after exercise. Similarly, Kamijo et al. (26) found that after exercising at moderate intensities, larger P3 amplitude was elicited compared with that after baseline (control) and vigorous exercise intensities, suggesting that exercise may be used to induce appropriate attentional allocation during performance of a cognitive task. Alternatively, research also supports that exercise at a moderate-intensity level induces higher contingent negative variation $(\mathrm{CNV})$ compared with that induced by light and vigorous exercise intensities. Because CNV is positively associated with attention and arousal level, this suggests that exercise may induce appropriate arousal when performing a cognitive task (24).

Although only a few studies have examined the effect of exercise for less than 20 min on cognition, our nonsignificant differences on cognitive performance after exercise for less than 20 min is consistent with previous findings. Yagi et al. (38) investigated the differences in visual and auditory oddball task performance as well as its elicited P3 after 10 min of exercise at light to moderate intensity; however, no exercise effects on either behavioral or neuroelectric indices were found. In addition, these results are consistent with the findings from the meta-analysis of Chang et al. (9), which indicated that when summarized across studies, the effects of exercise for less than 10 min on subsequent cognitive performance were negative. Our research adds to the knowledge base by examining a cognitive task involving both information processing and executive function and by testing the effects of duration on these aspects of cognitive performance. Thus, the results of this study in consideration with findings from previous research indicate that exercise performed for $10 \mathrm{~min}$ has no benefits for cognitive performance after exercise.

In contrast to findings from previous studies looking at the effects of exercise of "long" duration on Stroop test performance $(23)$ or short-term memory $(13,14,19)$, in this study, the 45 -min acute exercise session had no effect on cognition, as assessed by the Stroop test. Notably, the participants we recruited and the exercise duration we used in this study (i.e., healthy younger adults, $45 \mathrm{~min}$ ) were different from those used by Hogervorst et al. (23) (i.e., highly trained triathletes and $60 \mathrm{~min}$ ), Cian et al. (13), Cian et al. (14) (i.e., endurance-trained adults and 120 
min), and Grego et al. (19) (i.e., trained cyclists and $180 \mathrm{~min}$ ). Individuals with high levels of fitness (as used in the previous studies) may improve cognition with longer durations of exercise, whereas participants with normal fitness status (as used in this study) do not achieve these same benefits. This conclusion is also supported by findings that greater benefits from acute exercise are observed in individuals with high fitness (9) or highly trained older participants relative to those with low-to-moderate levels of fitness or those who are untrained (33). It should be noted that after prolonged exercise (i.e., $2 \mathrm{~h}$ ), even participants with a high level of fitness would be expected to experience decreases in cognitive performance possibly because of central fatigue associated with heat stress, dehydration, or hypoglycemia $(13,14,28)$ or with negative effects on information processing (19). In contrast, it is speculated that individuals with a lower level of fitness or less physical training may receive more beneficial cognitive effects from acute exercise of shorter duration. Hence, future studies will be necessary to identify the optimal intensity and duration combination for groups that differ in terms of cognitive abilities (e.g., relative to agerelated cognitive decline), fitness (e.g., in those with low vs those with high levels of fitness), or other variables that might conceivably moderate the relation between acute exercise and cognitive performance.

Some limitations of this study should be acknowledged and considered in interpreting these results. The study was focused exclusively on moderate exercise intensity. It is possible that intensity and duration must be considered simultaneously to understand the optimal effects on cognitive performance. In other words, although results of this study indicate that 20 min of exercise is optimal for the effects of moderate intensity on Stroop task performance, other durations may be optimal for light- or vigorous-intensity exercise. Another limitation of this study was the focus on a single measure of executive function. Executive function is a comprehensive cognitive construct that consists of several subcomponents of cognitive performance, namely shifting, updating, and inhibition. The Stroop test is considered to provide a measure of inhibition (32). Etnier and Chang (16) have argued that the effect of acute exercise on executive function might be dependent on the particular subcomponents of executive function assessed. Hence, future studies are necessary to expand our understanding of how duration and intensity work together to influence cognitive performance and to explore how effects are similar or different depending on the particular type of cognitive task. Furthermore, although the sample size was appropriate to statistically assess the beneficial effect of acute exercise on cognition on the basis of an a priori power analysis, because we limited our sample to right-handed collegeage men, future research with a larger and more heterogeneous sample will be necessary to assess the extent to which these findings apply to the broader population.

With regard to future research, it is important for researchers to expand on the behavioral findings of this study by including measures of potential mechanisms. By understanding mechanisms, we have the potential to dramatically advance our understanding of how acute exercise might be prescribed to benefit cognitive performance. For example, one might consider applying neuroeletric techniques such as electroencephalography to further our understanding in 
terms of the relation between exercise duration and underlying neuroelectric responses (e.g., event-related potential [ERP], CNV, spectral frequency) in cognition. Researchers might also assess neurotrophic factors such as brain-derived neurotrophic factor or catecholamines (e.g., dopamine, norepinephrine) because these biological mechanisms have also been implicated in research on acute exercise and cognitive performance $(17,20,37)$. It is particularly important that evidence of a relation between exercise duration and cognitive function has been demonstrated in the present study, providing the foundation to further investigate the underlying mechanism.

In conclusion, acute aerobic exercise for $20 \mathrm{~min}$ at a moderate intensity facilitates cognitive performance for both information processing and inhibition; however, this improvement was not observed when exercising for 10 or $45 \mathrm{~min}$. Although future research is required to replicate these findings, the present research has implications with regard to prescribing exercise to enhance cognition in healthy younger adults.

This research was funded by a portion of a grant from the Ministry of Science and Technology in Taiwan to Yu-Kai Chang (NSC 102-2918-1-179-001; NSC 102-2420-H-179-001-MY3).

All authors report no potential conflict of interest.

The results of the present study do not constitute endorsement by the American College of Sports Medicine.

\section{REFERENCES}

1. American College of Sports Medicine. ACSM's Guidelines for Exercise Testing and Prescription. 9th ed. New York (NY): Lippincott Williams and Wilkins; 2013. p. 42-180.

2. Arent SM, Landers DM. Arousal, anxiety, and performance: a reexamination of the inverted-U hypothesis. Res Q Exerc Sport. 2003; 74 (4): 436-44.

3. Barella LA, Etnier JL, Chang YK. The immediate and delayed effects of an acute bout of exercise on cognitive performance of healthy older adults. J Aging Phys Act. 2010; 18: 87-98.

4. Bauman A, Bull F, Chey T, et al. The International Prevalence Study on Physical Activity: results from 20 countries. Int J Behav Nutr Phys Act. 2009; 6 (21): 1-11.

5. Borg GA. Psychophysical bases of perceived exertion. Med Sci Sports Exerc. 1982; 14 (5): 377-81. 
6. Chang YK, Chu IH, Chen FT, Wang CC. Dose-response effect of acute resistance exercise on Tower of London in middle-aged adults. J Sport Exerc Psychol. 2011; 33 (6): 866-83.

7. Chang YK, Etnier JL. Exploring the dose-response relationship between resistance exercise intensity and cognitive function. J Sport Exerc Psychol. 2009; 31: 640-56.

8. Chang YK, Etnier JL, Barella LA. Exploring the relationship between exercise-induced arousal and cognition using fractionated response time. Res Q Exerc Sport. 2009; 80 (1): 78-86.

9. Chang YK, Labban JD, Gapin JI, Etnier JL. The effects of acute exercise on cognitive performance: a meta-analysis. Brain Res. 2012; 1453: 87-101.

10. Chang YK, Tsai CL, Huang CC, Wang CC, Chu IH. Effects of acute resistance exercise on cognition in late middle-aged adults: general or specific cognitive improvement? J Sci Med Sport. 2014; 17: 51-5.

11. Chang YK, Tsai CL, Hung TM, So EC, Chen FT, Etnier JL. Effects of acute exercise on executive function: a study with a Tower of London Task. J Sport Exerc Psychol. 2011; 33 (6): 847-65.

12. Chmura J, Nazar K, Kaciuba-Uscilko H. Choice reaction time during graded exercise in relation to blood lactate and plasma catecholamine thresholds. Int J Sports Med. 1994; 15 (4): $172-6$.

13. Cian C, Barraud PA, Melin B, Raphel C. Effects of fluid ingestion on cognitive function after heat stress or exercise-induced dehydration. Int J Psychophysiol. 2001; 42: 243-51.

14. Cian C, Koulmannn N, Barraud PA, Raphel C, Jimenez C, Melin B. Influences of variations in body hydration on cognitive function: effects of hyperhydration, heat stress, and exerciseinduced dehydration. J Psychophysiol. 2000; 14: 29-36.

15. Davranche K, Audiffren M. Facilitating effects of exercise on information processing. $\mathbf{J}$ Sports Sci. 2004; 22: 419-28.

16. Etnier JL, Chang YK. The effect of physical activity on executive function: a brief commentary on definitions, measurement issues, and the current state of the literature. J Sport Exerc Psychol. 2009; 31: 469-83.

17. Ferris LT, Williams JS, Shen CL. The effect of acute exercise on serum brain-derived neurotrophic factor levels and cognitive function. Med Sci Sports Exerc. 2007; 39 (4): 728-34.

18. Golding LA. YMCA Fitness Testing and Assessment Manual. Champaign (IL): Human Kinetics; 1989. p. 1-247.

19. Grego F, Vallier JM, Collardeau M, et al. Effects of long duration exercise on cognitive function, blood glucose, and counterregulatory hormones in male cyclists. Neurosci Lett. 2004; 364 (2): 76-80. 
20. Griffin EW, Mullally S, Foley C, Warmington SA, O’Mara SM, Kelly AM. Aerobic exercise improves hippocampal function and increases BDNF in the serum of young adult males. Physiol Behav. 2011; 104 (5): 934-41.

21. Hillman CH, Pontifex MB, Raine LB, Castelli DM, Hall EE, Kramer AF. The effect of acute treadmill walking on cognitive control and academic achievement in preadolescent children. Neuroscience. 2009; 159: 1044-54.

22. Hillman CH, Snook EM, Jerome GJ. Acute cardiovascular exercise and executive control function. Int J Psychophysiol. 2003; 48: 307-14.

23. Hogervorst E, Riedel W, Jeukendrup A, Jolles J. Cognitive performance after strenuous physical exercise. Percept Mot Skills. 1996; 83: 479-88.

24. Kamijo K, Nishihira Y, Hatta A, et al. Changes in arousal level by differential exercise intensity. Clin Neurophysiol. 2004; 115: 2693-8.

25. Kamijo K, Nishihira Y, Hatta A, et al. Differential influences of exercise intensity on information processing in the central nervous system. Eur J Appl Physiol. 2004; 92: 305-11.

26. Kamijo K, Nishihira Y, Higashiura T, Kuroiwa K. The interactive effect of exercise intensity and task difficulty on human cognitive processing. Int J Psychophysiol. 2007; 65 (2): 114-21.

27. Lambourne K, Tomporowski PD. The effect of exercise-induced arousal on cognitive task performance: a meta-regression analysis. Brain Res. 2010; 1341: 12-24.

28. Lieberman HR. Hydration and cognition: a critical review and recommendations for future research. J Am Coll Nutr. 2007; 26 (5 Suppl): 555S-61.

29. Liou YM, Jwo CJC, Yao KG, Chiang LC, Huang LH. Selection of appropriate Chinese terms to represent intensity and types of physical activity terms for use in the Taiwan version of IPAQ. J Nurs Res. 2008; 16 (4): 252-63.

30. MacLeod CM. Half a century of research on the Stroop effect: an integrative review. Psychol Bull. 1991; 109 (2): 163.

31. McMorris T, Graydon J. Effect of exercise on cognitive performance in soccer-specific tests. J Sports Sci. 1997; 15: 459-68.

32. Miyake A, Friedman NP, Emerson MJ, Witzki AH, Howerter A, Wager TD. The unity and diversity of executive functions and their contributions to complex "frontal lobe" tasks: a latent variable analysis. Cogn Psychol. 2000; 41: 49-100.

33. Pesce C, Cereatti L, Forte R, Crova C, Casella R. Acute and chronic exercise effects on attentional control in older road cyclists. Gerontology. 2011; 57 (2): 121-8.

34. Tomporowski PD. Effects of acute bouts of exercise on cognition. Acta Psychol (Amst). 2003; 112: 297-324. 
35. Verburgh L, Königs M, Scherder EJ, Oosterlaan J. Physical exercise and executive functions in preadolescent children, adolescents and young adults: a meta-analysis. Br J Sports Med. 2014; 48 (12): 973-9.

36. Wechsler D, Corporation P. WAIS-III: Administration and Scoring Manual: Wechsler Adult Intelligence Scale. New York (NY): The Psychological Corporation; 1997. p. 1-268.

37. Winter B, Breitenstein C, Mooren FC, et al. High impact running improves learning. Neurobiol Learn Mem. 2007; 87 (4): 597-609.

38. Yagi Y, Coburn KL, Estes KM, Arruda JE. Effects of aerobic exercise and gender on visual and auditory P300, reaction time, and accuracy. Eur J Appl Physiol Occup Physiol. 1999; 80 (5): 402-8. 